\title{
Product Replacement in the Monster
}

\author{
Petra E. Holmes, Stephen A. Linton, and Scott H. Murray
}

\section{CONTENTS}

1. Introduction

2. The Product Replacement Algorithm

3. Computing the Monster

4. Product Replacement in the Monster

5. Conclusions

Acknowledgments

References

2000 AMS Subject Classification: Primary 20-04, 20D08

Keywords: Monster group, randomised algorithms

\begin{abstract}
We show that the product replacement algorithm can be used to produce random elements of the Monster group. These random elements are shown to have the same distribution of element orders as uniformly distributed random elements after a small number of steps.
\end{abstract}

\section{INTRODUCTION}

Computing in finite groups often requires a supply of random elements. There are several known methods for producing them. The best known practical method is the product replacement algorithm which is given in Section 2.

The Monster is the largest of the 26 sporadic simple groups. It has order

808017424794512875886459904961710757005754368000000000

its minimal faithful permutation and matrix representation degrees are respectively 97239461142009186000 and 196882 . This makes it far harder to work with than the 25 smaller sporadics.

In Section 3, we describe techniques which allow limited computation in the Monster. In Section 4, we describe our experiments to assess the effectiveness of combining these techniques with a suitable version of the product replacement algorithm, and in Section 5, we present our results and conclusions.

\section{THE PRODUCT REPLACEMENT ALGORITHM}

Let $G$ be a finite group generated by the set $X$. The product replacement algorithm uses an array $s=$ $\left(s_{1}, \ldots, s_{m}\right)$ of elements of $G$ satisfying the property that $\left\langle s_{1}, \ldots, s_{m}\right\rangle=G$. We require that $m$ be larger than the size of $X$. Initially we take the entries in $s$ to be the elements of $X$, with repetitions to fill out the array. Then at each stage of the algorithm, we choose distinct random integers $i$ and $j$ between 1 and $m$; and then replace $s_{i}$ by the product $s_{i} s_{j}$. We then return the new value of $s_{i}$ as 
our random element. It is known from [Celler et al. 95] that the random elements returned converge in the long term to a fixed distribution. While this distribution is often close to uniform, [Pak 01] has shown that in certain cases, it can be very far from uniform.

A more recent variant of this algorithm [LeedhamGreen and Murray 02] does converge to the uniform distribution. In this variant, we have an extra group element $s_{0}$, called the accumulator. Then at each step we do product replacement as above, but in addition we choose a third random integer $k$ and multiply $s_{0}$ by $s_{k}$. Then $s_{0}$ is returned as our random element.

We have run 100 tests of the product replacement algorithm (with and without an accumulator) with different random seeds. We assess the randomness of the elements produced using the $\chi^{2}$ statistic at the 0.9 probability level, applied to a test value derived from the orders of the random elements. We consider that the algorithm has converged at step $t$ if, for at least nine out of the subsequent ten steps, the $\chi^{2}$ value is below the 0.9 level, as would be expected from genuinely random elements. These methods for testing product replacement are based on [Babai et al. 01, Celler et al. 95].

\section{COMPUTING IN THE MONSTER}

Computing in the Monster is very different to computing in smaller groups. In most of the groups in which we work, generating elements can be stored as permutations or matrices. In the Monster we must take a different approach, as seen in the three constructions of [Linton et al. 98], [Holmes and Wilson], and [Wilson 00]. The construction of [Linton et al. 98], which uses 3-local subgroups and linear algebra in characteristic 2, gives us the fastest way of computing in $\mathbb{M}$ and so we choose it for our computations.

All three of the constructions use three generators, two of which generate a local subgroup. In the construction of [Linton et al. 98], two of the generators, $C$ and $D$, generate a subgroup isomorphic to $3^{1+12} \cdot 2$ Suz: 2 , the normaliser of an element in class $3 B$. The third generator, $T$, normalizes a subgroup of $\langle C, D\rangle$ isomorphic to $3^{2+5+10}:\left(\mathrm{M}_{11} \times 2^{2}\right)$, the centraliser of two commuting elements of class $3 B$. The element $T$ extends this group to $3^{2+5+10}:\left(\mathrm{M}_{11} \times \mathrm{D}_{8}\right)$. The dimension 196882 module for $\mathbb{M}$ over $G F(2)$ restricts to $3^{1+12} \cdot 2 \mathrm{Suz}: 2$ with shape

$$
142 \oplus 32760 \oplus\left(3^{6} \otimes 90\right),
$$

where all dimensions are over $G F(4)$ except 142 . So $C$ and $D$ are represented as files each containing four matrices, one for each piece of the representation. Similarly, the module structure for $3^{2+5+10}:\left(\mathrm{M}_{11} \times \mathrm{D}_{8}\right)$ allows $T$ to be stored as a collection of small matrices.

There are two main programs: one which calculates the image of any vector in the 196882-dimensional space under any of the generators, and one which can multiply together the elements in the local subgroup and return the product in the same format as the generators. In this paper, we use the vector-image program to calculate orders of words in the generators.

It takes approximately $60 \mathrm{~ms}$ for each occurrence of $T$ in a word to multiply a vector by a word using a Pentium II $/ 450 \mathrm{MHz}$ processor with $384 \mathrm{MB}$ of RAM. This operation is about 100 times faster than when using the construction of [Holmes and Wilson], although that construction is the more frequently used as it gives easy access to 2-local subgroups and comes equipped with a method for shortening words [Holmes 02].

\section{PRODUCT REPLACEMENT IN THE MONSTER}

We performed 100 independent incarnations of the product replacement algorithm, both with and without an accumulator, running them for 20 steps with an accumulator and 25 without.

To assess the uniformity of the distribution of random elements that we obtained using the $\chi^{2}$ test, we need some easily calculated property of these elements which will take a reasonably small number of values and whose distribution in the whole Monster is known. The $\chi^{2}$ test can then be used to compare the distribution of the values which appear in our sample with the true distribution of the value. In the Monster, the only suitable property which it appears feasible to calculate is element order, which has also been used in studying product replacement in other groups [Celler et al. 95]. To meet the requirements of the $\chi^{2}$ test, that each outcome have expected frequency at least 1 for the sample size being used, we group the orders $\{1,2, \ldots, 23,25,28,33,34,44,45,55,105,110\}$ together to form a single test value.

In fact, we do not strictly use element order, but instead use the order of the action of the elements on a fixed test vector. It is highly likely, but not proven, that this vector lies in a faithful orbit of $\mathbb{M}$. Otherwise, there will be a very small chance of obtaining a divisor of the correct order. Since this would make the orders look less, rather than more, random, it does not invalidate our conclusions.

The product replacement algorithm was actually performed in the free group on three generators using an 


\begin{tabular}{lll} 
Number & \multicolumn{1}{c}{$\chi^{2}$} & \multicolumn{1}{c}{$\chi^{2}$} \\
of steps & with accumulator & no accumulator \\
1 & 670.051 & 419.923 \\
2 & 390.119 & 367.669 \\
3 & 249.562 & 300.125 \\
4 & 163.061 & 252.426 \\
5 & 142.792 & 247.926 \\
6 & 62.536 & 274.574 \\
7 & 65.525 & 223.413 \\
8 & $\mathbf{3 6 . 8 8 2}$ & 94.410 \\
9 & 50.313 & 131.215 \\
10 & 37.671 & 56.936 \\
11 & 37.680 & 76.733 \\
12 & 35.448 & $\mathbf{5 2 . 5 2 7}$ \\
13 & 57.899 & 35.812 \\
14 & 48.167 & 33.932 \\
15 & 37.387 & 38.222 \\
16 & 34.160 & 44.395 \\
17 & 37.068 & 43.748 \\
18 & 47.394 & 49.358 \\
19 & 30.610 & 40.715 \\
20 & 40.744 & 38.068 \\
21 & & 45.162 \\
22 & & 36.205 \\
23 & & 38.297 \\
24 & & 46.629 \\
25 & & 43.727
\end{tabular}

TABLE 1. $\chi^{2}$ values. The entries in bold show where convergence has occurred.

array of length 4, resulting, for each random seed, in two sequences of words (one obtained using the accumulator, and the other not). This part of the calculation and the computation of $\chi^{2}$ values was done in GAP [GAP 02].

A C program, using subroutines derived from [Linton et al. 98], was then used to compute the orders of these words evaluated at the three generators described in Section 3 .

\section{CONCLUSIONS}

Table 1 gives the $\chi^{2}$ values for each step. It can be seen that product replacement with an accumulator converges after 8 steps, and for product replacement, we need 12 .

One use of random element generation in any group is to obtain elements of specific conjugacy classes or orders, for use in subsequent calculations, such as finding standard generators [Wilson 96]. To assess the suitability of product replacement in the Monster for this purpose, we tabulate in Table 2 the number of steps of product replacement with an accumulator that we have to perform before we see elements of multiples of each possible order amongst our hundred runs. We are only interested in multiples of element orders because we can get the element of the required order by powering up. It is clear

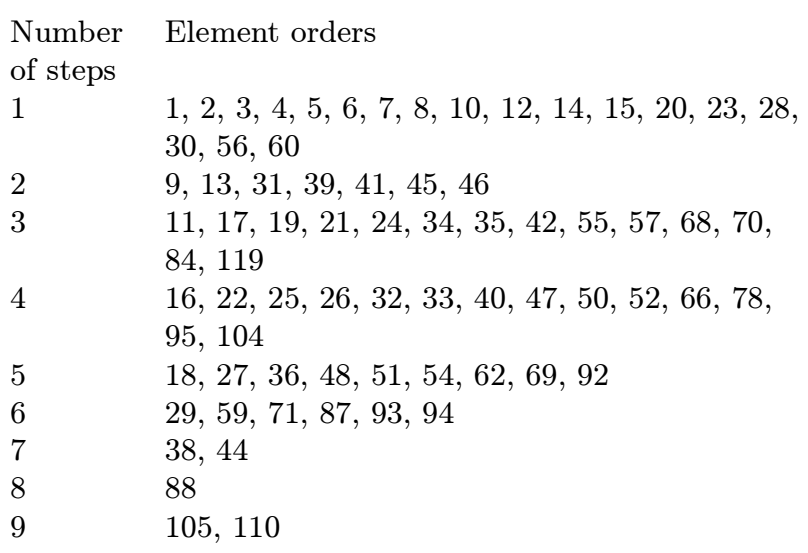

TABLE 2. Element orders occurring in the output of product replacement.

from the table that short words are not suitable for generating random elements as there are some orders of elements which we were unable to find without using many steps. To assess the cost of computing with random elements produced in this way, we graph in Figure 1 the length of the words generated against the number of steps of product replacement. Applying a word of length $n$ to

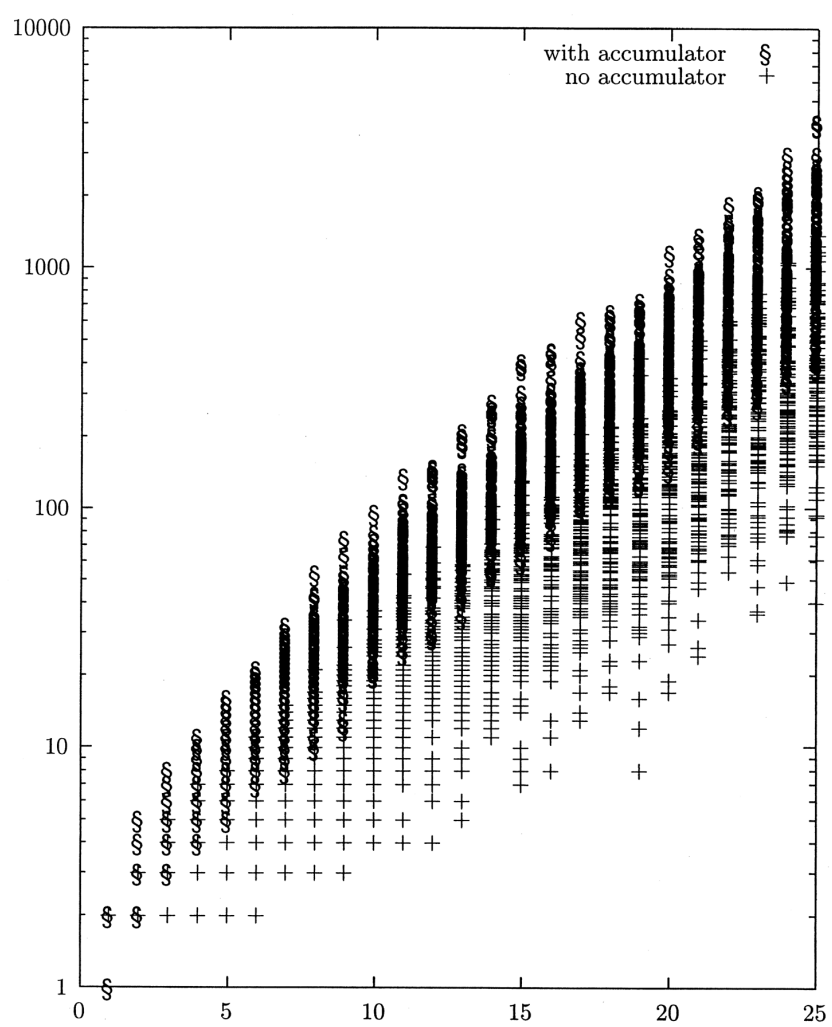

FIGURE 1. Word lengths for product replacement in the free group. 
a single vector takes about $60 \mathrm{nms}$ on a Pentium II/450 processor.

We conclude that, while it is not practical to use a single product replacement calculation to produce a series of random elements of the Monster as is done in other groups, since the words involved would rapidly grow too long, it would appear that, at least as far as element orders are concerned, starting a fresh product replacement algorithm and running it for between 8 and 15 steps (depending on the quality of randomness required) is both a feasible and an effective way to generate pseudorandom elements of the Monster.

Note that it is easy to see that the elements obtained in this way must be far from uniformly distributed on $\mathbb{M}$. By counting the possible random choices, we see that $n$ step product replacement with (without) accumulator can produce at most $(4 * 3 * 4)^{n}\left((4 * 3)^{n}\right)$ distinct outcomes, and this reaches the order of the Monster only for $n \geq 32(n \geq 49)$. What our experiments show is that product replacement approaches the same distribution of element orders as the uniform distribution of elements much sooner than this.

\section{ACKNOWLEDGMENTS}

The first author is supported by EPSRC grant GR/R95265/01. The computations described in this paper were performed on equipment provided by EPSRC grant GR/M32351/01.

\section{REFERENCES}

[Babai et al. 01] László Babai, Walter Kim, Scott H. Murray, and Rebecca Vessenes. "Quality of Random Elements in Finite Groups." Unpublished.

[Celler et al. 95] Frank Celler, Charles R. Leedham-Green, Scott H. Murray, Alice C. Niemeyer, and E. A. O'Brien. "Generating Random Elements of a Finite Group." Comm. Algebra 23 (1995), 4931-4948.

[Holmes 02] P. E. Holmes. "Computing in the Monster." PhD diss., Birmingham, 2002.

[Holmes and Wilson] P. E. Holmes and R. A. Wilson. "A New Computer Construction of the Monster Using 2Local Subgroups." J. LMS. To appear.

[Leedham-Green and Murray 02] C. R. Leedham-Green and Scott H. Murray. "Variants of Product Replacement." In Computational and Statistical Group Theory, pp. 97-104, Contemporary Mathematics 298, Providence, RI: American Mathematical Society, 2002.

[Linton et al. 98] S. A. Linton, R. A. Parker, P. G. Walsh, and R. A. Wilson. "Computer Construction of the Monster." J. Group Theory 1 (1998), 307-337.

[Pak 01] Igor Pak. "What Do We Know about the Product Replacement Algorithm?" In Groups and Computation, III (Columbus, OH, 1999), pp. 301-347. Berlin: de Gruyter, 2001.

[GAP 02] The GAP Group. GAP-Groups, Algorithms, and Programming, Version 4.3. Available from World Wide Web (http://www.gap-system.org), 2002.

[Wilson 96] R. A. Wilson. "Standard Generators for Sporadic Simple Groups." J. Algebra 184 (1996), 505-515.

[Wilson 00] R. A. Wilson. "A Construction of the Monster Group over GF(7), and an Application." Preprint, 2000.

Petra E. Holmes, School of Mathematics and Statistics, University of Birmingham, Edgbaston, Birmingham B15 2TT, United Kingdom (P.E.Holmes@bham.ac.uk)

Stephen A. Linton, School of Computer Science, University of St. Andrews, North Haugh, St. Andrews, Fife, KY16 9SS, United Kingdom (sal@dcs.st-and.ac.uk)

Scott H. Murray, Faculteit Wiskunde en Informatica, Technische Universiteit Eindhoven, Postbus 513, 5600 MB Eindhoven, The Netherlands (smurray@win.tue.nl)

Received June 12, 2002; accepted March 20, 2003. 